\title{
Redefining Quality Measurement in Cancer Care
}

\author{
Elizabeth A. Nardi, MS ; James McCanney, JD; Katy Winckworth-Prejsnar, MPHª; Alyssa A. Schatz, MSW ${ }^{a}$; \\ Kerin Adelson, $\mathrm{MD}^{\mathrm{b}}$; Marcus Neubauer, MDc; Mary Lou Smith, JD, MBA ${ }^{\mathrm{d}}$; \\ Ronald Walters, MD, MBA, MHA, MS ; and Robert W. Carlson, MD
}

\begin{abstract}
Quality measurement in oncology is increasing in significance as payment schemes shift from volume to value. As demand for quality measures increases, challenges in the development of quality measures, standardization across measures, and the limitations of health information technology have become apparent. Moreover, the time and financial burden associated with developing, tracking, and reporting quality measures are substantial. Despite these challenges, best practices and leaders in the field of quality measurement in oncology have emerged. To understand the current challenges and promising practices in quality measurement and to explore future considerations for measure development and measure reporting in oncology, NCCN convened the NCCN Policy Summit: Redefining Quality Measurement in Oncology. The summit included discussion of the current quality landscape and efforts to develop quality measures, use of quality measures in various programs, patient perspective of quality, and challenges and best practices for quality reporting.
\end{abstract}

J Natl Compr Canc Netw 2018;16(5):473-478 doi: $10.6004 /$ jnccn.2018.7028

\section{Executive Summary}

Quality measurement in oncology is an iterative process that continues to evolve. The importance of meaningful quality measures is increasing as payment schemes shift from volume to value. Further, there exists a need to standardize measures and minimize reporting burden. Accomplishing this requires health information technology (HIT) systems that are sophisticated enough to capture the right data and automate reporting of measures.

To understand the current challenges and promising practices in quality measurement and to explore future considerations for measure development and measure reporting in oncology, NCCN convened the NCCN Policy Summit: Redefining Quality Measurement in Oncology in Washington, DC, on September 25, 2017. Stakeholders gathered to discuss the challenges and opportunities for measuring quality care in oncology (see supplemental eAppendix 1, available with this article at JNCCN.org, for a list of participating speakers and panelists). The summit included discussion of the

\footnotetext{
aNational Comprehensive Cancer Network, Fort Washington, Pennsylvania; bSmilow Cancer Hospital and Yale Cancer Center, Yale School of Medicine, New Haven, Connecticut; 'McKesson Specialty Health, The Woodlands,

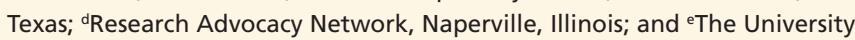
of Texas MD Anderson Cancer Center, Houston, Texas.

Submitted March 2, 2018; accepted for publication March 19, 2018.
}

current quality landscape and efforts to develop quality measures, use of quality measures in various programs, patient perspective of quality, and challenges and best practices for quality reporting. The program consisted of short presentations and roundtable discussions with lively dialogue and time for audience questions.

This article explores challenges and opportunities in measuring and reporting quality in oncology, considers future trends and shifts in quality measurement and reporting, and highlights the discussion and ideas addressed at the NCCN Policy Summit.

\section{The Quality Measure Landscape in Oncology}

The US healthcare system is rapidly moving toward paying for value. The passage, with bipartisan support, of the Medicare Access and CHIP Reauthorization Act of 2015 (MACRA) solidified this shift in payment for Medicare, and many private insurers are following. In

The authors have disclosed that they have no financial interests, arrangements, affiliations, or commercial interests with the manufacturers of any products discussed in this article or their competitors.

Correspondence: Alyssa A. Schatz, MSW, National Comprehensive Cancer Network, 275 Commerce Drive, Suite 300, Fort Washington, PA 19034 Email: schatz@nccn.org 
oncology specifically, examples of value-based payment models include financial incentives for adhering to clinical pathways, patient-centered medical homes, bundled payments, specialty accountable care organizations, and the Center for Medicare \& Medicaid Innovation's (CMMI) Oncology Care Model (OCM). ${ }^{1}$ As payment shifts from volume to value, measuring and reporting quality is at the forefront of the discussion among payers, policymakers, clinicians, and patients. In order to address current challenges and opportunities for measuring quality care, it is important to understand the evolution of the measurement of quality within oncology, as well as the larger healthcare system in the United States.

The quality measure landscape has progressed from one predominately focused on a providercentric measurement of value toward a more system-centric and an increasingly patient-centric approach. ${ }^{2}$ Historically, a provider-centric measurement of quality focused on training, education, certification, and processes of care. In a system-centric measurement approach, the focus does not exclude the importance of the provider, but is also on the entire system of care for patients looking more closely at cost, resource utilization, site of care, supportive infrastructure, and care coordination between providers. Patient-centric care emphasizes individual patient preferences, needs, and values to ensure patient values guide clinical decision-making. Recent years have seen growing recognition within healthcare systems that patient-centric care is required to achieve high-quality care for patients. ${ }^{2}$

Moving forward, there are several key requirements to fully implement system-centric and patientcentric measurements for reporting on quality care, including sophisticated and integrated data systems, multisystem analytics, transparency and interoperability of data, system attribution and accountability for outcomes (rather than exclusively provider), and recognition of the fluid nature of measures over time. ${ }^{2}$ Lastly, discrepancies between the perception of quality between the provider and patient, especially at time of diagnosis, require metrics and reporting measures to better align differences in treatment decisions.

\section{Incorporating Patient Perspectives in Quality Measurement of Cancer Care}

Patients, policymakers, institutions, payers, providers, and other stakeholders have various perceptions of quality care, as well as various metrics for quality in cancer care. From the patient perspective, there is an identified need to incorporate individual patient goals, expectations, and values to achieve patientcentric care. Mary Lou Smith, a patient advocate and co-founder of the Research Advocacy Network, views quality in 2 ways: quality of care and quality of treatment. ${ }^{3}$ Quality of care includes effective provider communication, tailored information and support, care coordination, and patient satisfaction. When looking at quality of treatment, the focus is on an intervention's effectiveness, adherence to guideline-concordant care, side effects, and burdens on the patient.

All patients want the best care possible, but context matters and the highest quality care might require tradeoffs based on proximity to treatment, socioeconomic status, type of cancer, age, stage of disease, time and disease process, side effects, and cost. $^{3}$ In other words, not all patients are the same and individual treatment goals may vary. Quality metrics should encompass ways to ask the patient their expectations, wishes, and values to ensure that the cancer care delivery system adequately meets the needs of patients, their families, and caregivers. Moving forward, the challenge for the oncology community is to quantify patients' expressed needs and desires about quality into defined metrics, for which data are collected and analyses are conducted, resulting in information that is comprehensible and actionable for the healthcare delivery system. ${ }^{4}$

\section{Measuring Quality in Oncology}

Quality measurement is an area of interest to all stakeholders; various cancer organizations lead efforts to develop, endorse, evaluate, validate, and understand measures for oncology. The Commission on Cancer (CoC), a program of the American College of Surgeons, establishes standards to ensure quality, multidisciplinary, and comprehensive cancer care delivery in healthcare settings. Cancer programs attain accreditation by reporting on these standards and participating in the National Cancer Database; $\mathrm{CoC}$ accreditation is nationally recognized by or- 
ganizations as having established data-driven performance measures for the provision of quality cancer care..$^{5}$ ASCO has developed an oncologist-led, practice-based quality assessment program designed to promote excellence in cancer care by helping practices create a culture of self-examination and improvement, known as the Quality Oncology Practice Initiative (QOPI). ${ }^{6}$ Practices can receive QOPI Certification, a 3-year certification that recognizes a practice's commitment to quality based on their performance on standards established in QOPI. The National Quality Forum (NQF), whose mission is to lead national collaboration to improve health and healthcare quality through measurement, conducts a rigorous process to endorse measures submitted by measure development organizations. NQF-endorsed measures are considered the gold standard for quality measurement; measures that are NQF-endorsed are the most frequently used in federal and private programs. ${ }^{7}$ The increasing importance of quality measures is apparent in the growing number of quality programs among stakeholders, including industry, federal and commercial payers, patient advocacy organizations, and professional societies.

Although the demand for quality reporting continues to increase, quality measure reporting in oncology faces significant and unique challenges, including the difficulties of data collection. Onerous reporting requirements have ballooned in recent years and are accompanied by significant cost. The Physicians Foundation, a nonprofit organization that examines critical issues affecting the US healthcare system by periodically surveying practicing physicians and patients, recently estimated that providers spend $\$ 15.4$ billion annually, or $\$ 40,069$ per physician per year, on quality reporting practices. ${ }^{8}$ Providers, health systems, and HIT organizations struggle to establish the data infrastructure to collect and analyze electronic health records (EHRs), claims, patient sourced, and analytic data. ${ }^{4}$ This highlights a need for various stakeholders in the medical community to work together to produce operationally feasible and standardized quality metrics and infrastructure. ${ }^{4}$

Precision medicine, with its promising implications for patient outcomes, also creates a unique challenge for quality measurement. As medical oncology becomes more precise with the advent of molecular testing and targeted therapies, the size of pa- tient populations in these cohorts narrows. At the same time, many recommend that oncology stakeholders work to make quality metrics more comprehensive and crosscutting. Successful quality metrics will be those that are not only backed by strong evidence, but also standardizable across practices to create national benchmarks. ${ }^{4}$

Clinical trials help drive quality measures by producing important data regarding expected or optimal outcomes. However, the overrepresentation of younger, healthier, better-educated people in clinical trials is a major hindrance to the ability of those responsible for quality measure creation when relying on the results for informing the treatment of older, sicker people. Payers are interested in quality metrics that rely on clinical trial results, but only to the extent that they are consistent with real-world outcomes. ${ }^{4}$ Incorporating real-world evidence into testing of measures for collection can help provide a basis for stronger quality measures, backed by actual real-world outcomes. ${ }^{4}$

A major gap in oncology quality measures is the dearth of patient-reported outcomes. More specifically, a patient-centric measurement of value should address patient preferences and values, the patient experience, patient engagement through shared decision-making, and patient outcomes based on patients' preferences and values. ${ }^{2}$ Patient perception of treatment positively correlates with survival rates and overall happiness. ${ }^{9}$ Therefore, oncologists must ensure patients understand all options and are active participants when delivering quality care. When it comes to translating these patient-centric aspects of care to quality measures, patients need to be involved in the development of metrics from the beginning.

The panel agreed that collaboration among a variety of oncology stakeholders toward meaningful and uniform measure development is critical to the implementation of successful quality measures across health systems. ${ }^{4}$ A partnership among a variety of oncology stakeholders to integrate varying data sources is needed to develop an inexpensive set of meaningful measures that works for providers, health systems, and most importantly, the patient.

\section{Highlighting Best Practices}

A common challenge to measuring quality is the lack of electronic, automatically reported measure- 
Nardi et al

ment. Although the goal is automated, standardized measures, most providers today struggle with numerous data sources, hard to abstract data points found in unstructured fields, and a large number of EHRs with various reporting mechanisms and capabilities. However, recent quality measure reporting capabilities have advanced significantly, due in large part to technological advances. Two particular best practices of quality measure reporting were highlighted at the NCCN Policy Summit: those of Yale Cancer Center and McKesson Specialty Health.

Dr. Kerin Adelson, MD, Chief Quality Officer, Yale Cancer Center/Smilow Cancer Hospital, provided insight into Yale Cancer Center's quality measurement efforts. In 2014, Dr. Adelson and colleagues undertook a project to better understand patterns of care in the last month of life for patients with cancer. The project was laborious and required manual chart validation in order to analyze 1 year of data; however, they were able to see gaps in care and identify areas for improvement. ${ }^{10}$ Being able to measure the percentage of patients admitted to an inpatient service, emergency department, or intensive care unit, or who received chemotherapy in the last 30 days of life was important to improve the quality of care being delivered. Through reducing harmful, unwanted care, Yale would improve quality and reduce cost. ${ }^{11}$

Yale collaborated with Flatiron Health (New York, NY), a healthcare technology and services company that is focused on accelerating cancer research and improving patient care by powering an innovative platform to address today's healthcare challenges. Flatiron uses both structured and unstructured data elements to build disease-specific outcomes databases. Specifically, Flatiron's business rules allow for detailed provider attribution that maps patients to specific providers and define a patient's primary oncologist. They also have robust date-ofdeath data, as well as staff resources and expertise to work with Yale's EHR data. Together, they have built and continue to develop a disease-specific outcomes database and an end-of-life utilization dashboard that allow for regional benchmarking within Yale's network as well as individual provider dashboards.

This technology enables Yale and its providers to view and analyze the care being delivered and performance on measures. Leveraging technology can help providers and systems improve the quality of care through process improvement plans. Further, investment in reusable infrastructure will allow for measurement over time and rapid development of new metrics. ${ }^{11}$

Dr. Marcus Neubauer, MD, Vice President and Medical Director, Payer and Clinical Services, McKesson Specialty Health and The US Oncology Network, provided a community oncology view of value-based care and different quality technologies. Supported by McKesson Specialty Health, The US Oncology Network unites the largest network of community oncologists in the United States. McKesson provides technology and clinical operations expertise supporting $>3,400$ oncologists, including roughly a third of practices participating in the OCM.

As new payment models and quality reporting have evolved over time, it has become clear that meeting these program requirements is untenable using paper record systems. McKesson has built a variety of technologies to support value-based care. In order to meet the demands of the OCM, The US Oncology Network's oncology-specific EHR, iKnowMed, has been updated with new structured fields and enhanced work flows, such as an automated treatment plan to be used for shared decision-making and fulfilling the core elements of the Institute of Medicine's Care Management Plan. Practice Insights, another McKesson technology platform, helps oncology practices by providing real-time quality measure feedback. By providing a dashboard view, providers can look at their performance and determine opportunities for improvement. Practice Insights also serves as a registry to submit data to the government, a crucial step in attesting to performance on quality metrics. Finally, McKesson's Clear Value Plus is a decision support tool for providers that highlights evidence-based treatment options and provides clinical and reimbursement information at the point of care. The software includes Value Pathways powered by NCCN and NCCN Clinical Practice Guidelines in Oncology (NCCN Guidelines), and allows for reporting on adherence to evidence-based guidelines, a component often included as a quality metric in value-based programs and contracts.

Both speakers agreed that provider use of technology advances value-based care and improves quality. Further, allowing providers to see and understand their performance is vital to quality improvement. 


\section{The Future of Quality Reporting in Cancer Care}

Providers and practices wishing to perform well in value-based payment models need to not only deliver high-quality care that is reflected by quality measures, but also be able to accurately demonstrate this through successful reporting of quality measures. Successful quality reporting requires alignment of many aspects of the healthcare system. HIT often plays a major role in measure reporting and can help to alleviate reporting burden for providers if measures are constructed in a way that can be automated. Dr. Adelson addressed 4 key requirements of HIT needed for successful quality reporting: the platform needs to accurately capture the appropriate data points for measure numerators and denominators; must be reproducible to ensure consistency of data; must be transparent, allowing providers to look at their performance and also understand the measure specifications; and must be continuous to be able to evaluate and give feedback on performance over time to drive meaningful change.

Quality measures for oncology often do not have recognized national benchmarks, leaving providers unsure of what is considered high performance. Similarly, measures with narrow specifications, such as percentage of adult patients (aged $\geq 18$ years) with metastatic colorectal cancer and KRAS gene mutation who were spared treatment with anti-EGFR monoclonal antibodies, ${ }^{12}$ may lead to small sample sizes depending on patient population. This presents particular challenges for rural practices with small patient populations that may not be able to meet the reporting requirements. Future measurement should look to develop crosscutting or general measures for oncology, and developers should work together to agree on measures that reflect high-quality cancer care.

Further, current quality measures for oncology are often criticized for focusing on processes rather than outcomes, although others argue that high-quality processes should lead to high-quality outcomes for patients with cancer. ${ }^{4}$ The hope is that sophisticated HIT platforms will be able to demonstrate such connection. Dr. Adelson and her colleagues at Yale are working to use the Flatiron database to study whether goals of care conversations are happening between providers and patients. First, they will determine whether natural language processing can be used to extract goals of care conversations; then they will determine the rate at which these conversations are occurring. High rates of goals of care conversations should then result in lower utilization at end of life among those providers. ${ }^{11} \mathrm{~A}$ focus on outcomes measures, or proven links from process to outcome, will help improve outcomes for patients with cancer.

Having different measures for different programs from different data sources creates an unsustainable reporting burden for providers. EHR systems frequently have more detailed, real-time information on a patient's treatment and care delivery, yet are often restricted to one system due to interoperability challenges. Claims data can provide all patient activity rather than being limited to one system. Increasingly, payers and providers are working together toward more automated systems for the future of quality reporting. Philadelphia, Pennsylvania has a health information exchange known as HealthShare Exchange that links the EHR systems of different hospital health systems and other healthcare providers with the claims data of healthcare insurers. ${ }^{13}$ Independence Blue Cross has created Facilitated Health Networks, designed to engage both the insurer and the healthcare provider to collaborate on matters such as shared accountability, the exchange of real-time data, and strengthening efficiency and coordination of patient care. ${ }^{14}$ These innovative collaborative efforts can reduce administrative burdens and allow payers to enter into more value-based arrangements to improve care for patients.

The panel acknowledges that quality measurement is an iterative process, and that measure development processes and priorities have evolved due to lessons learned. ${ }^{15}$ Quality in oncology is challenging to measure and unique from other medical specialties because the evolution of best practice is occurring rapidly. Increased data interoperability and automated systems, as well as standardized measurements that incorporate patient preferences, are key elements of successful quality measurement in oncology.

\section{Conclusions}

The US healthcare system is steadily moving toward value-based care, which inherently requires quality measurement. Measuring and reporting on quality, however, presents challenges. Lack of standardiza- 
Nardi et al

tion of measures in various programs leads to increasing reporting burden for providers. Disparate, incomplete data sources and evolving treatment paradigms are important factors to consider when developing new measures in oncology. Despite gaps and challenges, it is important to note that measurement has evolved and will continue to evolve to reflect true quality care. Moving forward, gaps such as incorporating patient preferences and values into measurement should be prioritized. Delivering high-quality care for patients with cancer is of utmost importance to ensuring optimal outcomes.

\section{References}

1. Deloitte. The evolution of oncology payment models: what can we learn from early experiments? 2016. Available at: https://www2.deloitte.com/ content/dam/Deloitte/us/Documents/life-sciences-health-care/us-lshcevolution-of-oncology-payment-models.pdf. Accessed April 27, 2018.

2. Walters R. The quality measure landscape in oncology. Presented at the NCCN Policy Summit: Redefining Quality Measurement in Oncology; September 25, 2017; Washington, DC.

3. Smith ML. Incorporating patient perspectives in quality measurement of cancer care. Presented at the NCCN Policy Summit: Redefining Quality Measurement in Oncology; September 25, 2017; Washington, DC.

4. Measuring quality in oncology: challenges, lessons learned, and the way forward. Panel discussion at the NCCN Policy Summit: Redefining Quality Measurement in Oncology; September 25, 2017; Washington, DC.

5. Commission on Cancer. Value and Benefits of Accreditation. Alexandria, VA: ASCO; October 17, 2017. Available at: https://www.facs.org/qualityprograms/cancer/coc/apply/benefitscoc. Accessed April 27, 2018.

6. ASCO. ASCO's Longstanding Commitment to Quality. October 17, 2017. Available at: https://connection.asco.org/magazine/features/ascolongstanding-commitment-quality

7. National Quality Forum. What We Do. Available at: https://www. qualityforum.org/what_we_do.aspx. Accessed April 27, 2018.

8. Casalino LP, Gans D, Weber W, et al. US Physician Practices Spend More Than \$15.4 Billion Annually to Report Quality Measures. Health Aff (Millwood) 2016;35:401-406.
9. Kimmel PL. Psychosocial factors in adult end-state renal disease patients treated with hemodialysis: correlates and outcomes. Am J Kidney Dis 2000;35:132-140.

10. Adelson KB, Velji S, Patel K, et al. Preparing for value-based payment: a stepwise approach for cancer centers. J Oncol Pract 2016;12:e924-932.

11. Adelson K, Neubauer M. Highlighting best practices in quality measurement and reporting in cancer care. Presented at the NCCN Policy Summit: Redefining Quality Measurement in Oncology; September 25, 2017; Washington, DC.

12. National Quality Forum. Patients with metastatic colorectal cancer and KRAS gene mutation spared treatment with anti-epidermal growth factor receptor monoclonal antibodies. Available at: http://www.qualityforum. org/QPS/1860. Accessed April 20, 2018.

13. HealthShare Exchange. About HSX. Available at: https://www. healthshareexchange.org/about-hsx. Accessed April 27, 2018.

14. Independence signs groundbreaking agreement with University of Pennsylvania Health System [new release]. Philadelphia, PA: Independence Newsroom; April 12, 2017. Available at: https://news. ibx.com/independence-signs-groundbreaking-agreement-universitypennsylvania-health-system/. Accessed April 27, 2018.

15. The future of quality reporting in cancer care. Panel discussion at the NCCN Policy Summit: Redefining Quality Measurement in Oncology; September 25, 2017; Washington, DC.

\section{See JNCCN.org for supplemental online content.}

